\title{
OPERATORS PRESERVING DETERMINANTS OF MATRICES OVER COMMUTATIVE RINGS
}

\section{XIANGUI ZHAO and GUOTING ZHANG}

Department of Mathematics

Huizhou University, Huizhou

Guangdong Province 516007

P. R. China

e-mail: zhaoxg@hzu.edu.cn

guoting.zhang@foxmail.com

\begin{abstract}
In this paper, we investigate entrywise operators that preserve determinants of matrices over commutative rings. We give necessary and sufficient conditions for entrywise operators to be determinant preserving in the full matrix space, the upper triangular matrix space, the symmetric matrix space, and the antisymmetric matrix space, respectively.
\end{abstract}

\section{Introduction}

Preserver problems is an active research area in matrix theory and operator theory. Those problems involve certain operators of matrix spaces that preserve certain properties of matrices (usually) over a field. For example, the linear preserver problem [8,9] involves characterizing operators on matrices that preserve some properties/invariants of matrices such as determinants [7], singularities [2], eignvalues [1], (local)

2010 Mathematics Subject Classification: 15A15, 15A86.

Keywords and phrases: determinant preserver, symmetric matrix space, triangular matrix space, anti-symmetric matrix space.

Received March 31, 2017

(C) 2017 Scientific Advances Publishers 
spectrum [3], etc.; Guillot and Rajaratnam [6] studied functions preserving positive definiteness for sparse matrices; Cao and Zhang [5] characterized additive rank-one preserving surjections on symmetric matrix spaces over a field of characteristic not 2 or 3 .

Let $R$ be a commutative ring with identity. An operator $f$ of the matrix space $M_{n}(R)$ is called an entrywise operator of $M_{n}(R)$ if there exists an operator $\tau$ of $R$ such that $f(A)=\left(\tau\left(a_{i j}\right)\right) \in M_{n}(R)$ for all $A=\left(a_{i j}\right) \in M_{n}(R)$. Note that an entrywise operator is not necessarily a linear operator. In this paper, we characterize entrywise operators that preserve determinants of matrices over a commutative ring. More precisely, we investigate entrywise operators preserving determinants of matrices in several matrix spaces: the full matrix space, the upper triangular matrix space, the symmetric matrix space, and the antisymmetric matrix space.

\section{Operators Preserving Determinants}

We first introduce several basic concepts that will be used later. Let $R$ be a commutative ring with identity, and $M_{n}(R)(n \geq 2)$ be the set of $n \times n$ matrices with entries from $R$. Let $T_{n}(R) \subset M_{n}(R)\left(\operatorname{resp} ., S_{n}(R) \subset\right.$ $\left.M_{n}(R), A S_{n}(R) \subset M_{n}(R)\right)$ be the subset consisting of all upper triangular (resp., symmetric, anti-symmetric) matrices. As usual, we use $0_{n}$ and $I_{n}$ to denote the zero matrix and identity matrix, respectively, in $M_{n}(R)$; use $A \oplus B$ to present the diagonal block matrix $\left(\begin{array}{cc}A_{m} & 0_{m n} \\ 0_{n m} & B_{n}\end{array}\right)$ $\in M_{m+n}(R)$.

Suppose $f$ is an operator of $R$, i.e., a mapping from $R$ to itself. Then $f$ extends to an entrywise operator of $M_{n}(R)$ by setting $f(A)=\left(f\left(a_{i j}\right)\right) \in$ $M_{n}(R)$ for any $A=\left(a_{i j}\right) \in M_{n}(R)$. Similarly, $f$ extends to an entrywise 
operator of $S_{n}(R)$. It is easy to see that $f$ extends to an entrywise operator of $T_{n}(R)$ if and only if $f(0)=0$; and $f$ extends to an entrywise operator of $A S_{n}(R)$ if and only if $f$ is an odd mapping, i.e., if and only if $f(0)=0$ and $f(-r)=-f(r)$ for all $r \in R$.

Determinants of matrices over $R$ are defined similarly to those over a field, i.e.,

$$
\operatorname{det} A=\sum_{\sigma \in S_{n}} \operatorname{sgn}(\sigma) a_{1 \sigma(1)} a_{2 \sigma(2)} \cdots a_{n \sigma(n)},
$$

where $A \in M_{n}(R)$ and $S_{n}$ is the set of permutations on the set $\{1,2, \ldots, n\}$. Determinants of matrices over a commutative ring share many properties with their counterpart over a field (see, e.g., [4]).

Definition 2.1. An operator $f$ of $R$ is said to preserves determinants in $M_{n}(R)\left(\operatorname{resp} ., T_{n}(R), S_{n}(R), A S_{n}(R)\right)$ if $f(\operatorname{det} A)=\operatorname{det} f(A)$ for all $A \in M_{n}(R)$ (resp., $A \in T_{n}(R), S_{n}(R), A S_{n}(R)$ ).

Note that in this paper we do not assume that $f$ is a linear operator.

The rest of this section is organized as follows. We first study (in Subsection 2.1) entrywise operators preserving determinants in matrix space $M_{n}(R)$. Then we characterize determinant preserving entrywise operators on symmetric matrix space $S_{n}(R)$ in Subsection 2.2. Finally, we give necessary and sufficient conditions for determinant preserving entrywise operators on anti-symmetric matrix space $A S_{n}(R)$ in Subsection 2.3.

\subsection{Matrix space and upper triangular matrix space}

Theorem 2.2. Let $R$ be a commutative ring with identity 1 and $f: R \rightarrow R$ be an operator of $R$ satisfying that there exists $u \in R$ such that $f(u)$ is a unit of $R$. Then $f$ preserves determinants in $M_{n}(R)(n \geq 2)$ if and only if $f=k \tau$, where $k \in R, k^{n-1}=1$ and $\tau$ is an automorphism of $R$. 
Proof. $(\Leftarrow)$ Suppose that $f=k \tau$, where $k \in R, k^{n-1}=1$ and $\tau$ is an automorphism of $R$. Then, for any $A=\left(a_{i j}\right) \in M_{n}(R)$, we have

$$
\begin{aligned}
\operatorname{det} f(A) & =\sum_{\sigma \in S_{n}} \operatorname{sgn}(\sigma) k \tau\left(a_{1 \sigma(1)}\right) \cdot k \tau\left(a_{2 \sigma(2)}\right) \cdots k \tau\left(a_{n \sigma(n)}\right) \\
& =k^{n} \tau\left(\sum_{\sigma \in S_{n}} \operatorname{sgn}(\sigma) a_{1 \sigma(1)} \cdot a_{2 \sigma(2)} \cdots a_{n \sigma(n)}\right) \\
& =f\left(\sum_{\sigma \in S_{n}} \operatorname{sgn}(\sigma) a_{1 \sigma(1)} \cdot a_{2 \sigma(2)} \cdots a_{n \sigma(n)}\right) \\
& =f(\operatorname{det} A) .
\end{aligned}
$$

$\Leftrightarrow)$ Suppose $f$ preserves determinants in $M_{n}(R)(n \geq 2)$, i.e.,

$$
f(\operatorname{det} A)=\operatorname{det} f(A), \text { for all } A \in M_{n}(R) .
$$

Particularly, $f\left(\operatorname{det} 0_{n}\right)=\operatorname{det} f\left(0_{n}\right)$, i.e., $f(0)=0$. Suppose $u \in R$ and $f(u)$ is invertible. Let $B=u \oplus I_{n-1} \in M_{n}(R)$. Then $f(u)=f(\operatorname{det} B)=\operatorname{det} f(B)$ $=f(u)[f(1)]^{n-1}$. Thus $[f(1)]^{n-1}=1$ and therefore $f(1)$ is invertible with $[f(1)]^{-1}=[f(1)]^{n-2}$.

$$
\begin{gathered}
\text { Let } C=\left(\begin{array}{ll}
a & 0 \\
0 & b
\end{array}\right) \oplus I_{n-2} \text {. Then it follows from Equation (1) that } \\
f(a b)=f(\operatorname{det} C)=\operatorname{det} f(C)=f(a) f(b)[f(1)]^{n-2} .
\end{gathered}
$$

Let $\tau=[f(1)]^{n-2} f$. Then we have that $\tau(a b)=\tau(a) \tau(b)$.

$$
\begin{aligned}
& \text { Let } D=\left(\begin{array}{cc}
a & -b \\
1 & 1
\end{array}\right) \oplus I_{n-2} \text {. Then we get from Equation (1) that } \\
& f(a+b)=f(\operatorname{det} D)=\operatorname{det} f(D)=f(a)-f(-b) .
\end{aligned}
$$


Hence, by letting $a=0$ in the above equation, we have $f(b)=f(0)-f(-b)$ $=-f(-b)$. Thus, by Equation (2), $f(a+b)=f(a)-f(-b)=f(a)+f(b)$, which implies $\tau(a+b)=\tau(a)+\tau(b)$. Therefore, $\tau$ is an automorphism of $R$ and $f=k \tau$ for $k=[f(1)]^{2-n}=f(1)$.

Note that, in the " $\Rightarrow$ " part of the proof, the condition "there exists $u \in R$ such that $f(u)$ is a unit of $R$ " was only used to deduct that $[f(1)]^{n-1}=1$ from the equation $f(u)=f(u)[f(1)]^{n-1}$. If we assume that $R$ is an integral domain and consider the equation $f(u)=f(u)[f(1)]^{n-1}$ in the filed of fractions of $R$, then the conditions " $f(u) \neq 0$ " and “ $f(u)=f(u)[f(1)]^{n-1 "}$ would imply $[f(1)]^{n-1}=1$. That is, we have the following:

Corollary 2.3. Let $R$ be an integral domain with identity 1 and $f: R \rightarrow R$ be an operator of $R$. Then $f$ preserves determinants in $M_{n}(R)(n \geq 2)$ if and only if one of the following holds:

(i) $f(r)=0$ for all $r \in R$;

(ii) $f=k \tau$, where $k \in R, k^{n-1}=1$ and $\tau$ is an automorphism of $R$.

By a similar argument to the proof of Theorem 2.2, we can prove the following theorem for upper triangular matrices.

Theorem 2.4. Let $R$ be a commutative ring with identity 1 and $f: R \rightarrow R$ be an operator of $R$ satisfying that there exists $u \in R$ such that $f(u)$ is a unit of $R$. Then $f$ preserves determinants in $T_{n}(R)(n \geq 2)$ if and only if $f=k \tau$, where $k \in R, k^{n-1}=1$ and $\tau(a b)=\tau(a) \tau(b)$ for all $a, b \in R$. 


\subsection{Symmetric matrix space}

Theorem 2.5. Let $R$ be a commutative ring with identity 1 such that $r^{2} \neq 0$ for all nonzero $r \in R$. Let $f: R \rightarrow R$ be an operator of $R$ satisfying that there exists $u \in R$ such that $f(u)$ is a unit of $R$. Then $f$ preserves determinants in $S_{n}(R)(n \geq 4)$ if and only if $f=k \tau$, where $k \in R, k^{n-1}=1$ and $\tau$ is an automorphism of $R$.

Proof. $(\Leftarrow)$ This direction follows from Theorem 2.2.

$(\Rightarrow)$ Suppose $f$ preserves determinants in $S_{n}(R)(n \geq 4)$. By a similar argument to the proof of Theorem 2.2, we get $f(0)=0,[f(1)]^{-1}=$ $[f(1)]^{n-2}$ and $\tau(a b)=\tau(a) \tau(b)$ for all $a, b \in R$, where $\tau=[f(1)]^{n-2} f$.

Hence, $f=k \tau$, where $k=[f(1)]^{2-n}=f(1)$ and $k^{n-1}=1$. It suffices to prove that $\tau(a+b)=\tau(a)+\tau(b)$ for all $a, b \in R$.

Let $A=\left(\begin{array}{lll}2 & 1 & 1 \\ 1 & 0 & 1 \\ 1 & 1 & 0\end{array}\right) \oplus I_{n-3} \in S_{n}(R)$. It is clear that det $A=0$. Thus $f(\operatorname{det} A)=0$, that is, $(2 f(1)-f(2))[f(1)]^{n-1}=0$. So we have that $f(2)=2 f(1)$, as $[f(1)]^{n-1}=1$. Let $B=\left(\begin{array}{cccc}2 & 1 & 1 & a+b \\ 1 & 0 & 1 & a \\ 1 & 1 & 0 & b \\ a+b & a & b & 0\end{array}\right) \oplus I_{n-4} \in S_{n}(R), a, b \in R$. It is easy to check that $f(\operatorname{det} B)=f(0)=0$ (note that $f(2)=2 f(1)$ ). Thus, we have

$$
0=f(\operatorname{det} B)=\operatorname{det} f(B)=[f(a)+f(b)-f(a+b)]^{2}[f(1)]^{2} \cdot[f(1)]^{n-4},
$$


which implies $[f(a)+f(b)-f(a+b)]^{2}=0$. By our assumption, $r^{2} \neq 0$ for all nonzero $r \in R$, so we get $f(a)+f(b)-f(a+b)=0$, i.e., $f(a+b)=$ $f(a)+f(b)$ for all $a, b \in R$. Therefore, $\tau(a+b)=\tau(a)+\tau(b)$ for all $a, b \in R$.

Particularly, if $R$ is an integral domain, we have the following:

Corollary 2.6. Let $R$ be an integral domain with identity 1 and $f$ be an operator of $R$. Then $f$ preserves determinants in $S_{n}(R)(n \geq 4)$ if and only if one of the following holds:

(i) $f(r)=0$ for all $r \in R$;

(ii) $f=k \tau$, where $k \in R, k^{n-1}=1$ and $\tau$ is an automorphism of $R$.

Proof. $(\Leftarrow)$ If $f$ is the zero mapping, clearly it preserves determinants for matrices in $S_{n}(R)$. If $f$ is in the case (ii), then the result follows from Theorem 2.5.

$\Leftrightarrow$ Since $R$ is an integral domain, $r^{2} \neq 0$ for all nonzero $r \in R$. Suppose $f$ is not the zero mapping, that is, $f(a) \neq 0$ for some $a \in R$. Then, by an argument as in Corollary 2.3, we can prove that $f$ satisfies condition (ii). This completes our proof.

\subsection{Anti-symmetric matrix space}

Recall that elements along the main diagonal of an anti-symmetric matrix are all zero. For any $A \in A S_{n}(R)$ with $n$ odd, $\operatorname{det} A=0$. An operator $f$ of $R$ is called an odd operator if $f(-r)=-f(r)$ for all $r \in R$ and $f(0)=0$.

Theorem 2.7. Suppose $R$ is a commutative ring with identity, $n$ is an odd integer, and $f$ is an operator of $R$. Then $f$ preserves determinants in $A S_{n}(R)$ if and only if $f$ is odd. 
Proof. It is clear.

It is easy to check the following lemma, which will be used in the proof for the case of even $n$.

Lemma 2.8. Suppose $R$ is a commutative ring, $n \geq 2$ is an even integer, and

$$
A=\left(\begin{array}{ccccc}
0 & x & x & \cdots & x \\
-x & 0 & x & \cdots & x \\
-x & -x & 0 & \cdots & x \\
\cdots & \cdots & \cdots & \cdots & \cdots \\
-x & -x & -x & \cdots & 0
\end{array}\right) \in A S_{n}(R)
$$

Then $\operatorname{det} A=x^{n}$.

Theorem 2.9. Suppose $R$ is a commutative ring with identity 1 satisfying that $r^{2} \neq 0$ for all nonzero $r \in R, n \geq 4$ is an even integer, and $f$ is an odd operator of $R$ such that $f(1)$ is a unit of $R$. Then $f$ preserves determinants if and only if $f=k \tau$, where $k \in R, k^{n-1}=1$ and $\tau$ is an automorphism of $R$.

Proof. Note that, since $f$ is odd, $f$ is an operator of $A S_{n}(R)$.

$(\Leftarrow)$ This direction follows from the " $\Leftarrow$ " part of the proof of Theorem 2.2 .

$(\Rightarrow)$ Suppose $f$ preserves determinants in $A S_{n}(R)$. Let

$$
A=\left(\begin{array}{cccc}
0 & 1 & \cdots & 1 \\
-1 & 0 & \cdots & 1 \\
\cdots & \cdots & \cdots & \cdots \\
-1 & -1 & \cdots & 0
\end{array}\right) \in A S_{n}(R)
$$


By Lemma $2.8, \operatorname{det} A=1$ and $\operatorname{det} f(A)=[f(1)]^{n}$. Thus $f(1)=f(\operatorname{det} A)=$ $\operatorname{det} f(A)=[f(1)]^{n}$, which implies $[f(1)]^{n-1}=1$ as $f(1)$ is invertible in $R$.

Let

$$
B=\left(\begin{array}{cccc}
0 & 0 & 1 & a \\
0 & 0 & b & a b \\
-1 & -b & 0 & 0 \\
-a & -a b & 0 & 0
\end{array}\right) \oplus\left(\begin{array}{cccc}
0 & 1 & \cdots & 1 \\
-1 & 0 & \cdots & 1 \\
\cdots & \cdots & \cdots & \cdots \\
-1 & -1 & \cdots & 0
\end{array}\right)_{n-4} \in A S_{n}(R), a, b \in R .
$$

Then, by easy calculation and Lemma 2.8, we have

$$
0=f(0)=f(\operatorname{det} B)=\operatorname{det} f(B)=-[f(1) f(a b)-f(a) f(b)]^{2}[f(1)]^{n-4} .
$$

Since, by assumption, $f(1)$ is invertible in $R$ and $r^{2} \neq 0$ for all nonzero $r \in R$, we obtain that $f(1) f(a b)-f(a) f(b)=0$. Letting $\tau=[f(1)]^{-1} f$, we have $\tau(a b)=\tau(a) \tau(b)$.

Not let

$$
C=\left(\begin{array}{cccc}
0 & -1 & 1 & a+b \\
1 & 0 & 1 & a \\
-1 & -1 & 0 & b \\
-a-b & -a & -b & 0
\end{array}\right) \oplus\left(\begin{array}{cccc}
0 & 1 & \cdots & 1 \\
-1 & 0 & \cdots & 1 \\
\cdots & \cdots & \cdots & \cdots \\
-1 & -1 & \cdots & 0
\end{array}\right)_{n-4} \in A S_{n}(R), a, b \in R .
$$

Direct calculation gives that

$$
0=f(\operatorname{det} C)=\operatorname{det} f(C)=[f(a+b)-f(a)-f(b)]^{2}[f(1)]^{n-2} .
$$

Hence, by a similar argument as above, we get $f(a+b)-f(a)-f(b)=0$, i.e., $f(a+b)=f(a)+f(b)$. Thus $\tau(a+b)=\tau(a)+\tau(b)$. Therefore, $\tau$ is an automorphism of $R$. 
Note that, for the case $n=2$, the above proof does not work. However, it is easy to see that an odd operator $f$ of a commutative ring $R$ preserves determinants in $A S_{2}(R)$ if and only if $f\left(a^{2}\right)=f(a) f(a)$ for all $a \in R$.

\section{Acknowledgement}

This work is supported in part by the NSF of Guangdong Province of China (2016A030310099, 2014A030310119), the Outstanding Young Teacher Training Program in Guangdong Universities (No. YQ2015155), the Start-Up Research Grant Program of Huizhou University (2015JB021), and the Scientific Research Innovation Team Project of Huizhou University (hzuxl201523).

\section{References}

[1] R. Alizadeh and F. Shakeri, Linear maps preserving Pareto eigenvalues, Linear and Multilinear Algebra 65(5) (2017), 1053-1061.

[2] P. Botta, Linear maps that preserve singular and nonsingular matrices, Linear Algebra and its Applications 20(1) (1978), 45-49.

[3] A. Bourhim and J. Mashreghi, Maps preserving the local spectrum of product of operators, Glasgow Mathematical Journal 57(03) (2015), 709-718.

[4] W. C. Brown, Matrices Over Commutative Rings, Marcel Dekker, Inc., 1993.

[5] Chong-guang Cao and Xian Zhang, Additive rank-one preserving surjections on symmetric matrix spaces, Linear Algebra and its Applications 362 (2003), 145-151.

[6] D. Guillot and B. Rajaratnam, Functions preserving positive definiteness for sparse matrices, Transactions of the American Mathematical Society 367(1) (2015), 627-649.

[7] J. Kalinowski, On preservers of determinant over a field, Demonstratio Mathematica 41(3) (2008), 519-524.

[8] Chi-Kwong $\mathrm{Li}$ and Stephen Pierce, Linear preserver problems, The American Mathematical Monthly 108(7) (2001), 591-605.

[9] Chi-Kwong Li and Nam-Kiu Tsing, Linear preserver problems: A brief introduction and some special techniques, Linear Algebra and its Applications 162 (1992), 217-235. 